\title{
Melanonychia Caused by Hydroxyurea in Hematologic Patients: Report of 4 Cases
}

\author{
Pektas SD1*, Pektas G² and Tetik Aydogdu C1 \\ ${ }^{1}$ Department of Dermatology, Mugla Sitki Kocman University, Turkey \\ ${ }^{2}$ Department of Hematology, Mugla Sitki Kocman University, Turkey
}

*Corresponding author: Suzan Demir Pektas, M.D., Department of Dermatology, Mugla Sitki Kocman University Faculty of Medicine, 48000, Mugla, Turkey, Tel: +90 252211 5219; Fax: +90 312 3116768; E-mail: suzandpektas@gmail.com

\section{Case Report \\ Volume 3 Issue 1}

Received Date: February 15, 2018

Published Date: March 08, 2018

DOI: $10.23880 /$ cdoaj-16000142

\section{Abstract}

Melanonychia is a nail pigmentation disorder which is predominantly seen in female patients treated by hydroxyurea; especially in patients with diagnosed myeloproliferative disorders. Hydroxyurea inhibits DNA synthesis through its action on ribonucleoside diphosphate-reductase, and can be lead to dermatological disorders including mucocutaneous side effects and nail disorders such as onycholysis, onychodystrophy, and melanonychia. The pathogenesis of hydroxyurea-induced melanonychia is not clear. We present the cases of 4 female patients that two of four patients with diagnosed as polycythaemia rubravera and two of four patients with diagnosed as essential thrombocytosis who are developed melanonychia during hydroxyurea therapy. It is emphasized that the side effects of chemotherapeutic drugs should asses carefully by observing the clinical historyin hematologic patients and treatment should modify, although the side effects will quickly disappear with the reduction or discontinuation of the drug.

Keywords: Melanonychia; Hydroxyurea; Polycythaemia Rubra; Essential Thrombocytosis

Abbreviations: HIV: Human Immunodeficiency Virus; CML: Chronic Myelogenous Leukemia.

\section{Case Report}

\section{Introduction}

Melanonychia is uncommon side effect of hydroxyurea that approximately $4.3 \%$ of the patients under hydroxyurea therapy can be affected [1]. Hydroxyurea inhibits DNA synthesis by blocking the M2 subunit of ribonucleotide reductase which is responsible for catalyzing the conversion of ribonucleotides to deoxyribonucleotides [2,3]. This is a cytostatic drug commonly used for management of myeloproliferative disorders and rarely in psoriasis and human immunodeficiency virus (HIV) infection treatment [2]. Case report of hydroxyurea-induced melanonychia are limited in literature $[1,3,4]$. Hydroxyurea therapy can caused different dermatologic disorders such as xerosis, lichenoid eruptions, alopecia, leg ulcers and hyperpigmentation, cutaneous vasculitis, palmar and plantar keratoderma, mucosal changes like oral ulcers, stomatitis, and hyperpigmentation, nail changes such as 
onycholysis, onychodystrophy, and melanonychia [1-4]. Although hydroxyurea induced nail pigmentation has been reported to occur as early as 4 months after the start of treatment, it is generally occurs after longer periods of therapy [5]. The pathogenesis of hydroxyurea-induced nail changes is not well understood, however several mechanisms have been suggested including a genetic predisposition, direct toxic effect of hydroxyurea on the nail bed and matrix and focal stimulation of melanocytes at the matrix level [5]. We reported 4 patients with melanonychia which occurs in the course of treatment with hydroxyurea treated at the department of hematology for the following diseases: two patients polycythaemia vera and two patients essential thrombocytosis).

\section{Case 1}

A 67-year-old female patient was admitted to our clinic with the complaint of color change in the hand nails for 15 days. She had been diagnosed with polisitemia rubra vera 6 months ago and was treated with hydroxyurea $1.5 \mathrm{gr} /$ day. The patient has had a history of hypertension for 10 years and has been taking amlodipine $10 \mathrm{mg}$ / day for 10 years. Our physical examination revealed diffuse pigmented bands of brownish-black hue of varying degrees of intensity,2-5 mm wide, involving all 10 nails on the hand nail (Figure 1). The bands on the fingernails were located proximal nail, because of the growth of nail. The nail plates and the proximal and lateral nail folds were normal except for the color changes. It is learned that the intensity of color changes on the fingernails was decreased after stopping hydroxyurea therapy. On other laboratory examinations were unremarkable.

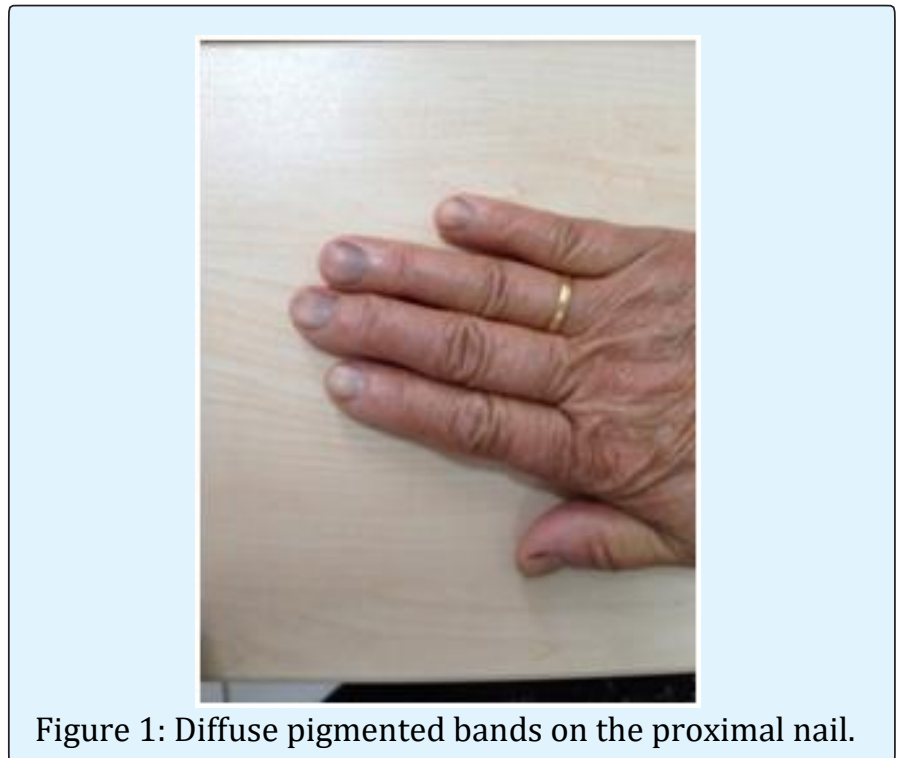

\section{Case 2}

A 74-year-old female patient presented with a color change in her fingers and toes. The patient was diagnosed with essential thrombocytosis 1 year ago. Treatment with hydroxyurea 1 gr / day was started. Complaints improved after 2 months of treatment. The patient, who had a history of hypertension for the past 18 years, has used valsartan $80 \mathrm{mg} /$ day and metoprolol $50 \mathrm{mg} /$ day. On physical examination revealed the transverse brownishblack pigmentation with different degrees of intensity on 20 nails of hands and feet (Figures 2a \& 2b). No other mucocutaneous abnormalities were observed. Laboratory examinations were unremarkable.

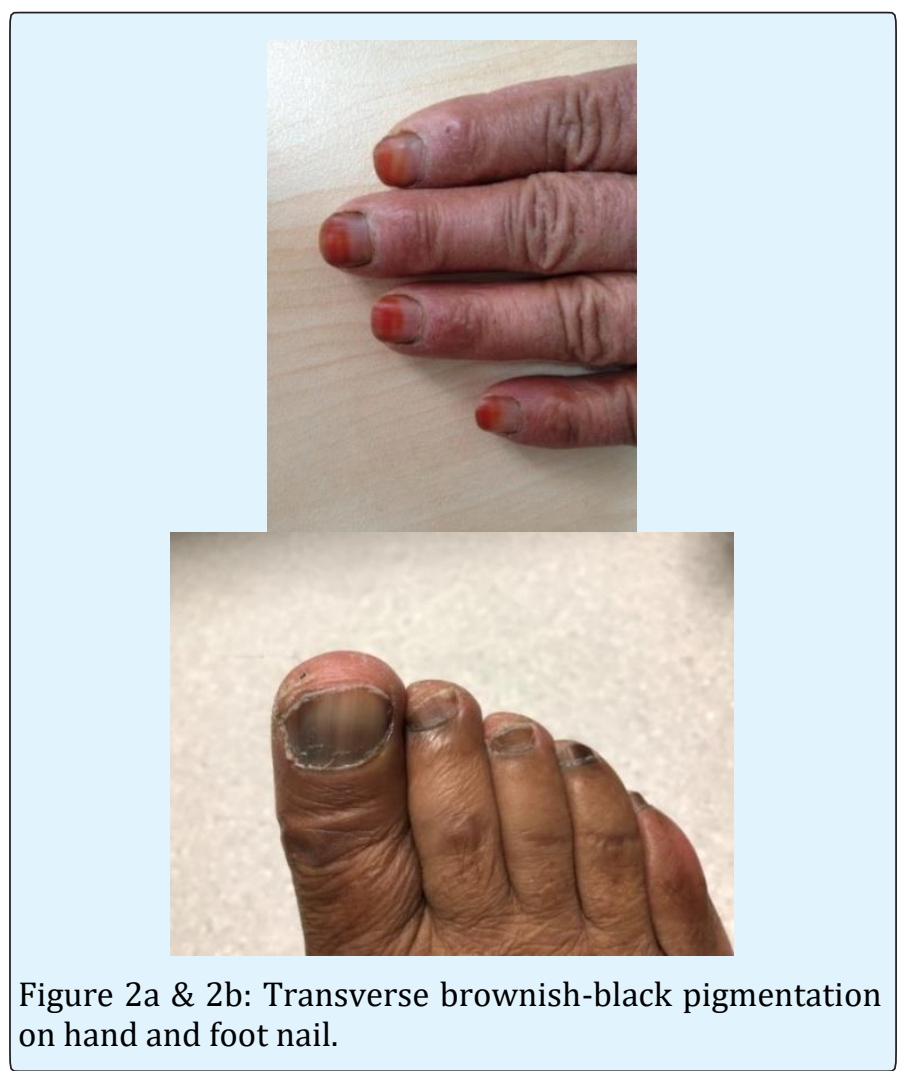

\section{Case 3}

A 62-year-old female patient presented with a color change in her hands fingers. The patient was diagnosed with essential thrombocytosis 18 months ago and started to treat with hydroxyurea. Treatment with hydroxyurea $0.5 \mathrm{gr} /$ day was started. Complaints improved after 8 months of treatment. The transverse brownish-black pigmentation and longitudinal bands on multiple nails of hands as well as kserotic skin were observed on dermatological examination (Figure 3). No other mucocutaneous abnormalities were observed. 


\section{Clinical Dermatology Open Access Journal}

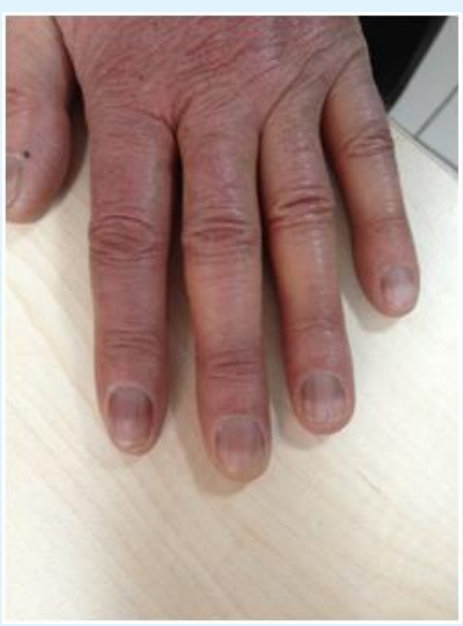

Figure 3: Transverse brownish-black pigmentation and longitudinal bands on multiple nails of hands.

\section{Case 4}

An 80 - year - old female patient presented with a 1 year complaint of color change on her fingers. The patient was diagnosed with polycythemia rubra vera 2 years ago and hydroxyurea $1.5 \mathrm{gr} /$ day treatment was started. The disease went into remission with the treatment. There is no known systemic disease and regular drug use. Our physical examination, diffuse pigmented bands of brownish-black hue of varying degrees of intensity 2-5 $\mathrm{mm}$ wide on the hand nails were identified (Figure 4). The bands on the fingernails were almost fully grown out and were located more distally, resembling a single band of transverse pigmentation. The nail plates and the proximal and lateral nail folds were normal. There was no drug history that could be associated with nail pigmentation.

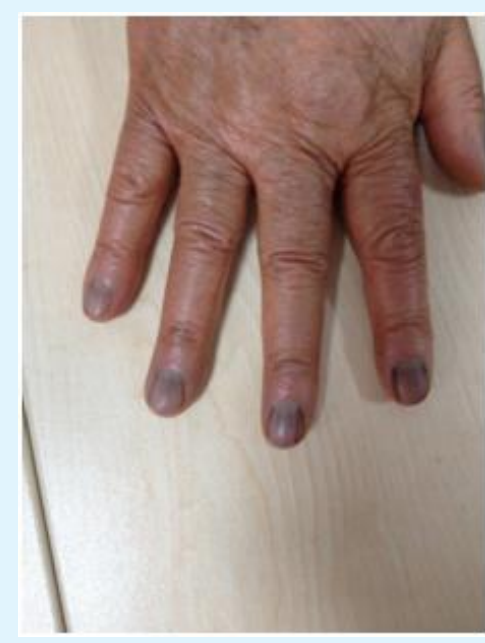

Figure 4: Diffuse pigmented bands on the hand nails.

\section{Discussion}

Melanonychia can be seen in some patients who are treated with long-term hydroxyurea [1]. Although the mechanism of melanonychia is not thoroughly understood, several mechanisms have been proposed including a genetic predisposition, photosensitisation, direct toxic effect of hydroxyurea on the nail bed and matrix, and focal stimulation of melanocytes at the matrix level $[5,6]$. It is more commonly seen among female [6]. In harmony with the published data in the literature, the all of cases with melanonychia were female who are under treatment with hydroxyurea at the department of hematology for polycythaemia vera and essential thrombocytosis.

Hydroxyurea used to treat in many of disorders including chronic myelogenous leukemia (CML), polycythemia vera, essential thrombocythemia, essential thrombocytosis and sickle cell anemia. It is usually well tolerated. Nevertheless, $13 \%$ of patients during long-term treatments present reversible and dose-dependent side effects. It is described different mucocutaneous abnormalities such as diffuse hyperpigmentation in the skin or mucosas, painful leg ulcers, alopecia, xerosis, lichenoid eruptions, squamous cell carcinomas, acral erythema, dermatomyositis-like syndromes and melanonychia [3]. The case report of hydroxyureainduced melanonychia is rare in the literature [1]. It is proposed that the time interval between the start of therapy and the first signs of nail pigmentation depend on the rate of nail growth; this finding would explain the later and less frequent appearance of changes in the toenails [1]. Therefore, it is reported that the onset of the melanonychia varies from 10 weeks to 5 years after initiation of the hydroxyurea [6]. The onset of the melanonychia in four cases varies from 2 months to 1 years after initiation of the hydroxyurea in accordance with the literature.

Hydroxyurea induced melanonychia was diagnosed based on their medical history and clinical examination. The differential diagnosis of hydroxyurea induced melanonychia can be supposed as subungual melanoma, nail matrix melanocytic nevus, lentigo, and lentiginoses of various types, other drug-induced pigmentation, pigmented squamous cell carcinoma, ethnic nail pigmentation, repetitive trauma-induced pigmentation, and subungual hemorrhages [6]. In clinical examination of hydroxyurea induced melanonychia can be observed nail pigmentation changes include longitudinal bands, transverse bands, and diffuse darkening as patterns of nail involvement [1]. 
Despite occuring multiple patterns in hydroxyureainduced melanonychia, simultaneously; longitudinal bands are the most common pattern, especially in female patients [1,7]. Transverse melanonychia seems to be related to the intermittent courses of chemotherapy because of a direct toxic effect on the nail matrix [1]. The thumb and index finger nails are involved, most frequently.

Consistent with other reports in the literature, our patients's melanonychia developed longitudinal pattern and monochromic, stable size and affect multiple nail, especially fingernail more than toenail. Moreover, our patients reported that their melanonychia developed after hydroxyurea therapy.

The other laboratory results of four patients were assessed as normal. When medical history and clinical findings of our patients examined, it was evaluated that nail changes could be attributed to hydroxyurea. It is demonstrates that an iatrogenic (drug-induced) cause should be considered in the case of longitudinal melanonychia affecting multiple nails [8].

If it is necessary, the hydroxyurea therapy can be continued, even at maximum dosage, as mentioned before. The necessity of stopping the therapy should be discussed, carefully. In our cases, hematologist decided to continue treatment with the same hydroxyurea dose used before in all patients by evaluating the risk-benefit ratio.

In conclusion, physicians who manage the hydroxyurea treatment should be aware of the different common and uncommon cutaneous reactions that may be anticipated in each particular patient. A correct diagnosis is essential to preventing undue fears in the patient.

\section{References}

1. Aste N, Fumo G, Contu F, Aste N, Biggio P (2002) Nail pigmentation caused by hydroxyurea: Report of 9 cases. J Am Acad Dermatol 47(1): 146-147.

2. Serrano-Falcón C, Pugnaire MA, Serrano-Falcón Mdel M, Morales MG, Serrano-Ortega S (2011) Toxicoderma caused by hydroxyurea. Int J Dermatol 50(11): 1435-1437.

3. Issaivanan M, Mitu PS, Manisha C, Praveen K (2004) Cutaneous manifestations of hidroxyurea therapy in childhood: casereport and review. Pediatr Dermatol 21(2): 124-127.

4. Salmon-Ehr V, Leborgne G, Vilque JP, Potron G, Bernard P (2000) Secondary cutaneous effects of hydroxyurea: prospective study of 26patients from a dermatologic consultation. Rev Med Interne 21(1): 30-34.

5. Teo RY, Tan E (2006) A case of hydroxyureainduced transverse melanonychia. Int $\mathrm{J}$ Dermatol 45(11): 1329-1330.

6. Karanth SS, Gupta A, Prabhu M (2014) Melanonychia and mucocutaneous hyperpigmentation from hydroxyurea 4 usefor the treatment of essential Thrombocytosis. Singapore Med J 55(1): e7-e8.

7. Zargari O, Kimyai-Asadi A, Jafroodi M (2004) Cutaneous Adverse Reactions to Hydroxyurea in Patients with Intermediate Thalassemia. Pediatric Dermatology 21(6): 633-635.

8. Nguyen AL, Körver JE, Theunissen CC (2017) Longitudinal melanonychia on multiple nails induced by hydroxyurea. BMJ Case Rep. 